\section{Transmit Precoding for Flat-Fading MIMO Multiuser Systems With Maximum Ratio Combining Receivers}

\author{
Adem Coskun, Student Member, IEEE, and Çag̃atay Candan
}

\begin{abstract}
We examine the application of transmit precoding in multiuser multi-input-multi-output (MIMO) communication systems with maximum ratio combining (MRC) receivers. In many multiuser applications, the maximum-likelihood or minimum mean-square error (MMSE) receivers can be prohibitive to implement due to their high implementation complexity. We examine the performance of the system with simple MRC receivers and carefully selected precoders, which are designed to compensate the lack of high-complexity receivers, at the transmitter side. We examine the sum MSE minimization and signal-to-interference-plus-noise ratio (SINR) balancing frameworks for the selection of precoders. The performance of two frameworks with MRC receivers are compared between themselves as well as with their counterparts implementing MMSE receivers. It has been observed that the SINR balancing framework with simple MRC receivers has little performance loss in comparison with the MMSE receivers with a proper selection of precoders.
\end{abstract}

Index Terms-Downlink, maximum ratio combining (MRC), minimum mean-square error (MMSE) receivers, multi-input-multi-output (MIMO) multiuser downlink, MIMO systems, signal-to-interference-plus-noise ratio (SINR) balancing.

\section{INTRODUCTION}

Multi-input-multi-output (MIMO) communication techniques are the key technologies enabling high data rate and reliable communications. Single-user MIMO systems have been studied in depth, and various techniques have been proposed to improve the data rate and to combat channel impairments such as fading and interference [1]. Multiuser MIMO systems have some additional challenges. On the uplink side, the channels between each user and the base station are different from each other. The base station can use the channel information of each user to estimate the transmitted bits by adapting any one of the single-user MIMO receiver architectures, such as minimum mean-square error (MMSE), zero forcing, successive interference cancellation, and maximum-likelihood (ML) decoding. On the downlink side, the base station simultaneously provides service to a group of users with possibly different quality-of-service requirements. Simultaneous service results in multiple-access interference (MAI) at the receivers. MAI cannot be effectively mitigated by a receiveronly operation, since in the downlink problem, signal and interference travel in the same channel. Different from uplink, the multiuser downlink communication problem necessitates indirect collaboration of receivers and the base station for interference mitigation. In the downlink problem, interference travels along with the signal of interest from the base station to each user. Indirect collaboration necessitates that the users should feed their channel information back to the transmitter and that the transmitter initiates a series of calculations using the channel-state information of each user for the selection of

Manuscript received February 10, 2010; revised August 18, 2010 and October 12, 2010; accepted November 16, 2010. Date of publication December 3, 2010; date of current version February 18, 2011. The review of this paper was coordinated by Prof. W. A. Krzymień.

A. Coskun is with the Applied DSP and VLSI Research Group, University of Westminster, London W1W 6UW, U.K. (e-mail: adem@alptron. com).

Ç. Candan is with the Department of Electrical and Electronics Engineering, Middle East Technical University, Ankara 06531, Turkey (e-mail: ccandan@metu.edu.tr).

Digital Object Identifier 10.1109/TVT.2010.2096484 appropriate precoding and, if needed, decoding vectors for the signal transmission.

In this paper, we focus on multiuser MIMO downlink communication systems where each user receives a single stream of data. In the absence of MAI, the maximal ratio combining (MRC) receiver is known to be the signal-to-noise ratio (SNR) maximizing receiver [2]. When MAI is present, higher complexity receivers such as MMSE or ML receivers are preferred for MAI mitigation [3], [4]. Implementing MMSE or ML receivers significantly increases the hardware cost, particularly for systems with many users. In this paper, we examine the performance degradation if MRC receivers are used instead of higher complexity alternatives in the presence of transmitter precoding. We show that the performance degradation can be insignificant if precoding vectors are accordingly selected. Hence, it can be said that the proposed method reduces the cost of receiver hardware at the expense of transmitter-side operations.

The main idea is to make good use of channel-state information at the transmitter side by selecting precoders that reduce MAI at the receiver end. If MAI can be significantly suppressed, simple MRC receivers, which are known to be optimum for white noise channels, can yield a good performance.

In the literature, single-stream multi-input-single-output (MISO) multiuser downlink systems have been studied in [5], and the multistream MIMO counterpart has been examined in [3] and [6]-[8], where the precoding vectors were linear. The nonlinear precoding schemes are given in [9] and [10]. In [5], the receivers are designed for a single stream of data, and in [7], [8], and [11], the receivers use optimized decoding vectors (different from MRC vectors) to estimate the transmitted bits. To the best of our knowledge, the topic of this paper, i.e., the performance loss of multiuser MIMO systems with simple MRC receivers in the presence of transmit precoding, has not been studied in the literature. The closest line of work that we are aware of is the application of the code-matched filter receiver in singleinput-single-output multiuser code division multiple access systems under additive noise and flat-fading channel conditions in the presence of transmit precoding [12], [13].

We present two different optimization frameworks for the selection of precoders. The first one optimizes the precoding vectors in the mean-square error (MSE) sense. The second framework optimizes the worst-case signal-to-interference-plus-noise ratio (SINR) among all users. The second framework is an extension of the work of Schubert and Boche [5], where a max-min optimization scheme is given for the MISO systems. Here, we extend the SINR balancing method of Schubert and Boche to MIMO systems equipped with MMSE or MRC receivers.

This paper is organized as follows: Section II describes the problem of precoder selection with MRC receivers. Sections III and IV present the proposed solutions and their performance comparison, respectively. Finally, this paper concludes with further discussions and comments.

\section{PROBLEM DESCRIPTION}

The proposed methods are described for a two-user system, each equipped with two antennas. This discussion can be easily generalized to the case of arbitrary number of users having arbitrary number of antennas.

We assume that a transmitter with $N_{t}$ antennas is serving two users under flat-fading channel conditions. The vector $\mathbf{h}_{m, k}^{T}$ is a row vector of dimension $1 \times N_{t}$ whose entries are the channel coefficients between the $k$ th antenna of user $m$, and each one of the $N_{t}$ antennas at 


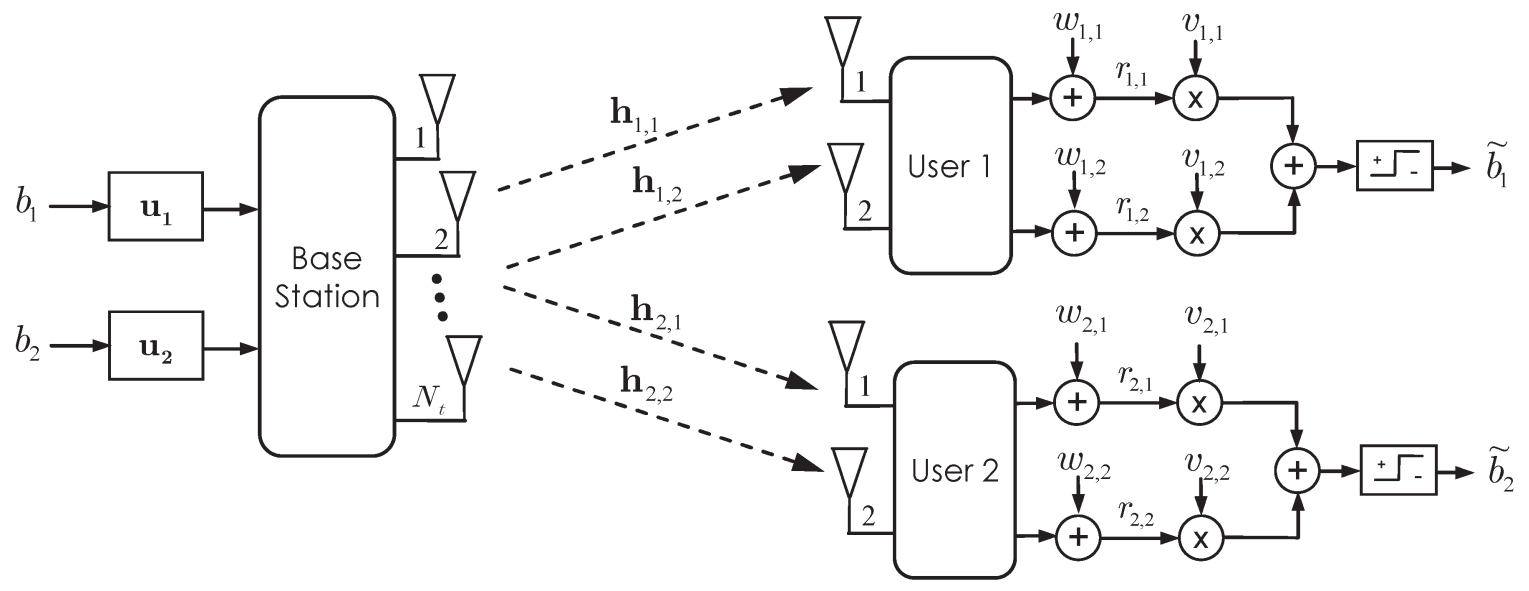

Fig. 1. System with two users, each equipped with two antennas.

the transmitter. $(\cdot)^{T}$ denotes vector transpose. The channel coefficients are assumed to be independent and complex Gaussian distributed with zero mean and unit variance. The signal received by the $k$ th antenna of user $m$ is written as follows:

$$
r_{m, k}=\mathbf{h}_{m, k}^{T} \mathbf{u}_{m} b_{m}+\underbrace{\sum_{n=1, n \neq m}^{K} \mathbf{h}_{m, k}^{T} \mathbf{u}_{n} b_{n}}_{\text {interference }}+w_{m, k} .
$$

In the preceding equation, $b_{m}$ denotes a symbol from the unit-variance constellation, i.e., $E\left\{\left|b_{m}\right|^{2}\right\}=1$, that is transmitted to the $m$ th user, and $\mathbf{u}_{m}$ is the associated precoding vector for its transmission; $w_{m, k}$ is the complex Gaussian distributed noise, which is independent and identically distributed for $m$ and $k$ with $\sigma_{w}^{2}$ variance; and $K$ is the number of users. The transmitter has a maximum power constraint, which can be expressed as $\sum_{m}\left\|\mathbf{u}_{m}\right\|^{2}=P_{\text {tot }}$, where $P_{\text {tot }}$ represents the total available power on the transmitter side.

The operation of a multiuser MIMO downlink system can be summarized follows: The transmitter sends a training sequence to let users estimate the channel between themselves and the base station. Users feed back the channel information to the transmitter. Given the channel information of all users, the transmitter selects the precoding vectors according to an optimization criterion. Then, the transmitter initiates a second phase of channel estimation using the selected precoding vectors. During this phase, users estimate the channel once more, but now, the channel estimate is the effective channel that is formed after the selection of precoding vectors. Under the described conditions, the effective channel should be $\widetilde{\mathbf{h}}_{m, k}^{T}=\mathbf{h}_{m, k}^{T}\left[\mathbf{u}_{1}, \ldots, \mathbf{u}_{K}\right]$. Here, $\widetilde{\mathbf{h}}_{m, k}^{T}$ denotes the effective channel formed when the precoding vectors $\left\{\mathbf{u}_{1}, \ldots, \mathbf{u}_{K}\right\}$ are used for a $K$-user system. The second phase of channel estimation allows users to indirectly learn the precoding vectors selected by the transmitter. After the completion of the training, the transmission of the data bits starts.

For a system with two users equipped with two antennas, as shown in Fig. 1, we have the following relation between the transmitter and receivers:

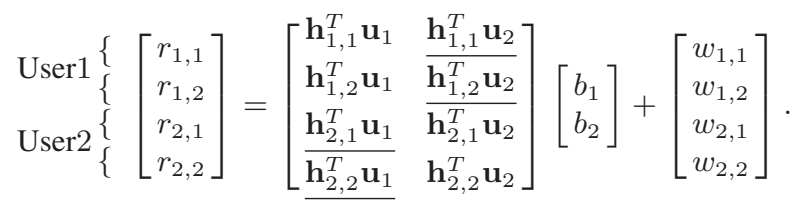

The terms causing interference at each reception are underlined. The precoding goal is the selection of $\mathbf{u}_{1}$ and $\mathbf{u}_{2}$ so that the interference terms are eliminated as much as possible.
As suggested in [3] and [6], given a large number of transmitting antennas, it is possible to find $\mathbf{u}_{1}$ orthogonal to both $\mathbf{h}_{2,1}$ and $\mathbf{h}_{2,2}$. Hence, $\mathbf{u}_{1}$ can be adjusted so that it does not cause any interference to the second user. For a general system, in order for the precoding vector $\mathbf{u}_{1}$ not to cause any interference to other users, it should be orthogonal to all $\mathbf{h}_{m, k}$ for $m \neq 1$. Therefore, for the vector $\mathbf{u}_{1}$ not to cause any interference, $N_{t}$ should be greater than the gross sum of antennas at all users. The described interference-free transmission is only applicable when the number of antennas at the transmitting side is exceptionally large. Pan et al. [14] have relaxed the constraint on the number of antennas by utilizing decoding matrices jointly optimized with the precoding vectors.

In addition to the interference-free communication, two other classes of methods have been proposed in the literature. In the first class, all users are assumed to implement MMSE receivers, and the sum of MSE of all users is minimized with a proper selection of precoding vectors [8]. In the second class, the worst SINR level among all users is maximized [5]. Different from the joint-MMSE technique, this technique allows balancing of SINR levels across the users and can also be used to serve different quality-of-service streams to the users.

In this paper, we extend the work of joint-MMSE minimization and SINR balancing framework by examining the performance degradation of the system when the receivers can only implement MRC.

We derive the set of equations satisfied by the optimal precoding vectors when MRC receivers are used. The equations for the optimal coefficients, in the case of MRC receivers, are nonlinear and complex. Here, our goal is to present the equations and motivate the usage of the iterative algorithms described in the next section for their solution. The signal received by the first user can be written as

$$
\mathbf{r}_{1}=\mathbf{H}_{1} \mathbf{u}_{1} b_{1}+\mathbf{H}_{1} \mathbf{u}_{2} b_{2}+\mathbf{w}
$$

where $\mathbf{H}_{1}$ is the channel matrix that corresponds to the first user, which is formed by concatenating channel gain vectors for the first user, i.e.,

$$
\mathbf{H}_{1}=\left[\begin{array}{l}
\mathbf{h}_{1,1}^{T} \\
\mathbf{h}_{1,2}^{T}
\end{array}\right]
$$

An MRC receiver combines the received signal using the relation $\widehat{b}_{1}=\mathbf{u}_{1}^{H} \mathbf{H}_{1}^{H} \mathbf{r}_{1}$. This leads to the following estimator:

$$
\widehat{b}_{1}=\underbrace{\left(\mathbf{u}_{1}^{H} \mathbf{H}_{1}^{H} \mathbf{H}_{1} \mathbf{u}_{1}\right)}_{\alpha} b_{1}+\underbrace{\left(\mathbf{u}_{1}^{H} \mathbf{H}_{1}^{H} \mathbf{H}_{1} \mathbf{u}_{2}\right)}_{\beta} b_{2}+\mathbf{u}_{1}^{H} \mathbf{H}_{1}^{H} \mathbf{w} .
$$


The SINR value after the MRC operation can then be written as

$$
\begin{aligned}
& \mathrm{SINR}=\frac{E\left\{|\alpha|^{2}\left|b_{1}\right|^{2}\right\}}{E\left\{|\beta|^{2}\left|b_{2}\right|^{2}+\left|\mathbf{u}_{1}^{H} \mathbf{H}_{1}^{H} \mathbf{w}\right|^{2}\right\}}
\end{aligned}
$$

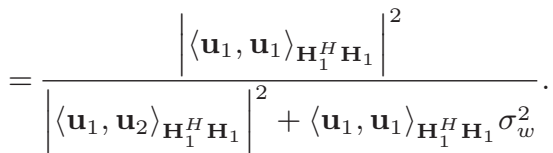

In the preceding equation, $\left\langle\mathbf{u}_{1}, \mathbf{u}_{2}\right\rangle_{\mathbf{H}_{1}^{H} \mathbf{H}_{1}}$ is a scalar that corresponds to the inner product of $\mathbf{u}_{1}$ and $\mathbf{u}_{2}$ with $\mathbf{H}_{1}^{H} \mathbf{H}_{1}$ weighting, i.e., $\left\langle\mathbf{u}_{1}, \mathbf{u}_{2}\right\rangle_{\mathbf{H}_{1}^{H} \mathbf{H}_{1}}=\mathbf{u}_{1}^{H} \mathbf{H}_{1}^{H} \mathbf{H}_{1} \mathbf{u}_{2}$. Here, the superscript $(\cdot)^{H}$ represents the Hermitian transpose. The SINR relation for the second user can be similarly written.

An optimization criterion is to maximize the SINR value of the users under a quality-of-service guarantee. To maximize the SINR value of the first user, irrespective of the second user's SINR, one can select $\mathbf{u}_{2}$ to be orthogonal to $\mathbf{u}_{1}$ in the $\mathbf{H}_{1}^{H} \mathbf{H}_{1}$ inner product space. However, this would only benefit the first user and provides no good to the MAI problem of the second user. In the following section, the SINR balancing method of Schubert and Boche is adapted to present a solution to this problem.

A second optimization goal is to select the precoding vectors such that the sum of MSE errors for two users is minimized. The MSE for the $k$ th user is given as $J_{k}\left(\mathbf{u}_{1}, \mathbf{u}_{2}\right)=E\left\{\left|b_{k}-\widehat{b}_{k}\right|^{2}\right\}$, where $\widehat{b}_{k}$ for $k=1$ is given in (5), and $E\{\cdot\}$ is the expectation operation. Taking the gradient of $J_{1}+J_{2}$ with respect to $\mathbf{u}_{1}$ and $\mathbf{u}_{2}$ gives the set of equations in (7), shown at the bottom of the page, whose solution produces the optimal set of precoding vectors.

The equation set should be solved under the power constraint $\left\|\mathbf{u}_{1}\right\|^{2}+\left\|\mathbf{u}_{2}\right\|^{2}=P_{\text {tot }}$ to avoid the trivial solution. The equations for the sum-MSE optimal precoding vectors utilizing MRC receivers are nonlinear and difficult to solve. We suggest the iterative algorithms presented in the next section for the solution.

\section{Proposed Methods}

In this section, we describe two different precoding techniques for multiuser MIMO systems, where each user receives a single data stream. The first technique adapts the method proposed in [8] to MRC receivers, and the second technique extends the MISO method proposed in [5] to MIMO receivers implementing MMSE or MRC receivers.

\section{A. SUm-MSE Optimization}

MMSE Receivers: In this framework, the goal of the transmitter is to select the precoders such that $E\left\{\sum_{k=1}^{2}\left|b_{k}-\widehat{b}_{k}\right|^{2}\right\}$ is minimized. Here, $\widehat{b}_{k}$ is the MMSE estimate of $b_{k}$, which can be written as follows:

$$
\left[\begin{array}{l}
\widehat{b}_{1} \\
\widehat{b}_{2}
\end{array}\right]=\underbrace{\left[\begin{array}{cccc}
v_{1,1} & v_{1,2} & 0 & 0 \\
0 & 0 & v_{2,1} & v_{2,2}
\end{array}\right]}_{\mathbf{G}}
$$

$$
\times(\underbrace{\left[\begin{array}{c}
\mathbf{h}_{1,1}^{T} \\
\mathbf{h}_{1,2}^{T} \\
\mathbf{h}_{2,1}^{T} \\
\mathbf{h}_{2,2}^{T}
\end{array}\right]}_{\mathbf{H}} \underbrace{\left[\begin{array}{ll}
\mathbf{u}_{1} & \mathbf{u}_{2}
\end{array}\right]}_{\mathbf{U}}\left[\begin{array}{c}
b_{1} \\
b_{2}
\end{array}\right]+\left[\begin{array}{l}
w_{1,1} \\
w_{1,2} \\
w_{2,1} \\
w_{2,2}
\end{array}\right])
$$

where $v_{m, k}$ is the combining weights for the $k$ th antenna of the $m$ th user. The reception equation can be also written as

$$
\widehat{\mathbf{b}}=\mathbf{G H U b}+\widetilde{\mathbf{w}}
$$

where $\mathbf{b}=\left[\begin{array}{ll}b_{1} & b_{2}\end{array}\right]^{T}$, and $\widetilde{\mathbf{w}}$ is a $2 \times 1$ vector whose entries are zero mean and have the covariance matrix of $\sigma_{w}^{2} \mathbf{G G}^{\mathbf{H}}$.

In the foregoing equation, the rows of a $\mathbf{G}$ matrix correspond to the MMSE receivers decoding each stream that is transmitted to the users. The rows of $\mathbf{G}$ can be calculated using standard MMSE formulation when precoding vectors are fixed to a set of vectors [1].

For a fixed set of decoding vectors, i.e., for a given $\mathbf{G}$ matrix, it can be shown that the optimal $\mathbf{U}$ minimizing sum-MSE as well as satisfying the power constraint $\operatorname{Tr}\left\{\mathbf{U}^{H} \mathbf{U}\right\}=P_{\text {tot }}$, is the solution of

$$
\mathbf{H}^{H} \mathbf{G}^{H}=\mathbf{H}^{H} \mathbf{G}^{H} \mathbf{G H U}+\mu \mathbf{U}
$$

where $\mu$ is Lagrange multiplier to satisfy the power constraint [8]. Then, the optimal U matrix can be written as follows:

$$
\mathbf{U}=\left(\mathbf{H}^{H} \mathbf{G}^{H} \mathbf{G H}+\mu \mathbf{I}\right)^{-1} \mathbf{H}^{H} \mathbf{G}^{H} .
$$

The precoder design is completed with the solution of $\mu$ from the power constraint, as shown in the following equation:

$$
\sum_{k=1}^{N_{t}} \frac{\lambda_{k}}{\left(\lambda_{k}+\mu\right)^{2}}=P .
$$

In this equation, $\lambda_{k}$ is the $k$ th eigenvalue of the matrix $\mathbf{H}^{H} \mathbf{G}^{H} \mathbf{G H}$.

The optimal decoding and precoding matrices can be iteratively found by fixing either $\mathbf{G}$ or $\mathbf{U}$ and optimizing over the other one until convergence [8]. The convergence of this iterative method is guaranteed since, at every iteration, the cost function is reduced, and the cost is lower bounded by 0 .

MRC Receivers: When users implement MRC instead of MMSE, the problem formulation significantly changes. The problem becomes a nonquadratic function of precoding vectors, as previously noted. In this case, the iterative optimization over $\mathbf{G}$ and $\mathbf{U}$ matrices does not have a cost monotonically decreasing with ongoing iterations. That is, the $\mathbf{G}$ matrix update given in Table I for MRC receivers is not guaranteed to reduce the sum-MSE at every iteration. Therefore, the convergence of sum-MSE formulation with MRC receivers is not guaranteed. In the following section, we compare the performance of sum-MSE formulation with MMSE and MRC receivers and discuss its convergence properties. The details of the proposed scheme is summarized in Table I.

$$
\left[\begin{array}{cc}
2\left(-1+\sigma_{w}^{2} / 2+\left\langle\mathbf{u}_{1}, \mathbf{u}_{1}\right\rangle_{\mathbf{H}_{1}^{H} \mathbf{H}_{1}}\right) \mathbf{H}_{1}^{H} \mathbf{H}_{1}, & \left\langle\mathbf{u}_{1}, \mathbf{u}_{2}\right\rangle_{\mathbf{H}_{1}^{H} \mathbf{H}_{1}} \mathbf{H}_{1}^{H} \mathbf{H}_{1}+\left\langle\mathbf{u}_{2}, \mathbf{u}_{1}\right\rangle_{\mathbf{H}_{2}^{H} \mathbf{H}_{2}} \mathbf{H}_{2}^{H} \mathbf{H}_{2} \\
\left\langle\mathbf{u}_{2}, \mathbf{u}_{1}\right\rangle_{\mathbf{H}_{2}^{H} \mathbf{H}_{2}} \mathbf{H}_{2}^{H} \mathbf{H}_{2}+\left\langle\mathbf{u}_{1}, \mathbf{u}_{2}\right\rangle_{\mathbf{H}_{1}^{H} \mathbf{H}_{1}} \mathbf{H}_{1}^{H} \mathbf{H}_{1}, & 2\left(-1+\sigma_{w}^{2} / 2+\left\langle\mathbf{u}_{2}, \mathbf{u}_{2}\right\rangle_{\left.\mathbf{H}_{2}^{H} \mathbf{H}_{2}\right)}\right) \mathbf{H}_{2}^{H} \mathbf{H}_{2}
\end{array}\right]\left[\begin{array}{l}
\mathbf{u}_{1} \\
\mathbf{u}_{2}
\end{array}\right]=\left[\begin{array}{c}
\mathbf{0} \\
\mathbf{0}
\end{array}\right]
$$


TABLE I

SUM-MSE MINIMIZATION FRAMEWORK

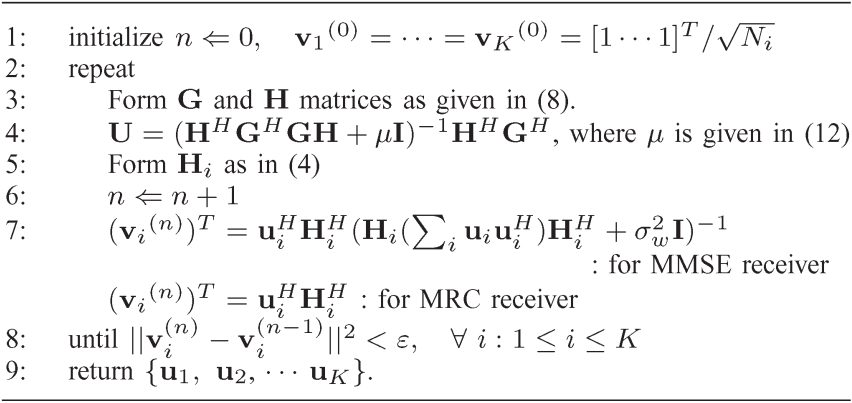

\section{B. SINR Balancing}

The following is an extension SINR balancing algorithm described in [5]. The extension optimizes the worst-case SINR among all users having multiple antennas.

MMSE Receivers: Denoting the decoding vector used by the $m$ th user with $\mathbf{v}_{\mathbf{m}}$, estimates for the information bits can be written as

$$
\left[\begin{array}{l}
\widehat{b}_{1} \\
\widehat{b}_{2}
\end{array}\right]=\underbrace{\left[\begin{array}{cc}
\mathbf{v}_{1}^{T} & \mathbf{0} \\
\mathbf{0} & \mathbf{\mathbf { v } _ { 2 } ^ { T }}
\end{array}\right]\left[\begin{array}{l}
\mathbf{h}_{1,1}^{T} \\
\mathbf{h}_{1,2}^{T} \\
\mathbf{h}_{2,1}^{T} \\
\mathbf{h}_{2,2}^{T}
\end{array}\right]}_{\widetilde{\mathbf{H}}}\left[\begin{array}{ll}
\mathbf{u}_{1} & \mathbf{u}_{2}
\end{array}\right]\left[\begin{array}{l}
b_{1} \\
b_{2}
\end{array}\right]+\underbrace{\left[\begin{array}{l}
\mathbf{v}_{1}^{T} \\
\mathbf{v}_{2}^{T}
\end{array}\right]\left[\begin{array}{l}
w_{1,1} \\
w_{1,2} \\
w_{2,1} \\
w_{2,2}
\end{array}\right]}_{\widetilde{\mathbf{w}}} .
$$

As in sum-MSE formulation, we assume that the decoding vectors are fixed and the optimization is carried over the precoding vectors first. If we denote the rows of $\widetilde{\mathbf{H}}$ with $\widetilde{\mathbf{h}}_{1}$ and $\widetilde{\mathbf{h}}_{2}$, (13) can be rewritten as follows:

$$
\left[\begin{array}{c}
\widehat{b}_{1} \\
\widehat{b}_{2}
\end{array}\right]=\left[\begin{array}{cc}
\widetilde{\mathbf{h}}_{1}^{T} \mathbf{u}_{1} & \widetilde{\mathbf{h}}_{1}^{T} \mathbf{u}_{2} \\
\widetilde{\mathbf{h}}_{2}^{T} \mathbf{u}_{1} & \widetilde{\mathbf{h}}_{2}^{T} \mathbf{u}_{2}
\end{array}\right]\left[\begin{array}{c}
b_{1} \\
b_{2}
\end{array}\right]+\left[\begin{array}{c}
\widetilde{w}_{1} \\
\widetilde{w}_{2}
\end{array}\right] .
$$

Note that, in (14), $\widetilde{w}_{1}$ and $\widetilde{w}_{2}$ denote the samples of white noise with variances $\left\|\mathbf{v}_{1}\right\|^{2} \sigma_{w}^{2}$ and $\left\|\mathbf{v}_{2}\right\|^{2} \sigma_{w}^{2}$, respectively. One can also say that, when decoding vectors are fixed, the equation system given in (13) reduces to a MISO system in (14). The output SINR for the first user can then be written as

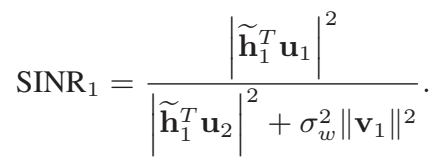

The SINR relation present in (15) is in the form that can be used and utilized by the max-min optimization of Schubert and Boche. The SINR balancing method assigns precoding vectors and their power to maximize the worst-case SINR for the multiuser MISO system given in (15).

Alternatively, when precoding vectors are fixed and the decoding vector $\mathbf{v}$ is allowed to vary, the output SINR for the first user can be written as follows:

$$
\operatorname{SINR}_{1}=\frac{\mathbf{v}_{1}^{T}\left(\mathbf{H}_{1} \mathbf{u}_{1} \mathbf{u}_{1}^{H} \mathbf{H}_{1}^{H}\right) \mathbf{v}_{1}^{*}}{\mathbf{v}_{1}^{T}\left(\mathbf{H}_{1} \mathbf{u}_{2} \mathbf{u}_{2}^{H} \mathbf{H}_{1}^{H}+\sigma_{w}^{2} \mathbf{I}\right) \mathbf{v}_{1}^{*}} .
$$

The $\mathrm{SINR}_{1}$ value given in (16) is maximized when $\mathbf{v}_{1}$ is selected as the generalized eigenvector of $\left(\mathbf{H}_{1} \mathbf{u}_{1} \mathbf{u}_{1}^{H} \mathbf{H}_{1}^{H}, \mathbf{H}_{1} \mathbf{u}_{2} \mathbf{u}_{2}^{H} \mathbf{H}_{1}^{H}+\sigma_{w}^{2} \mathbf{I}\right)$
TABLE II

SINR BALANCING FRAMEWORK

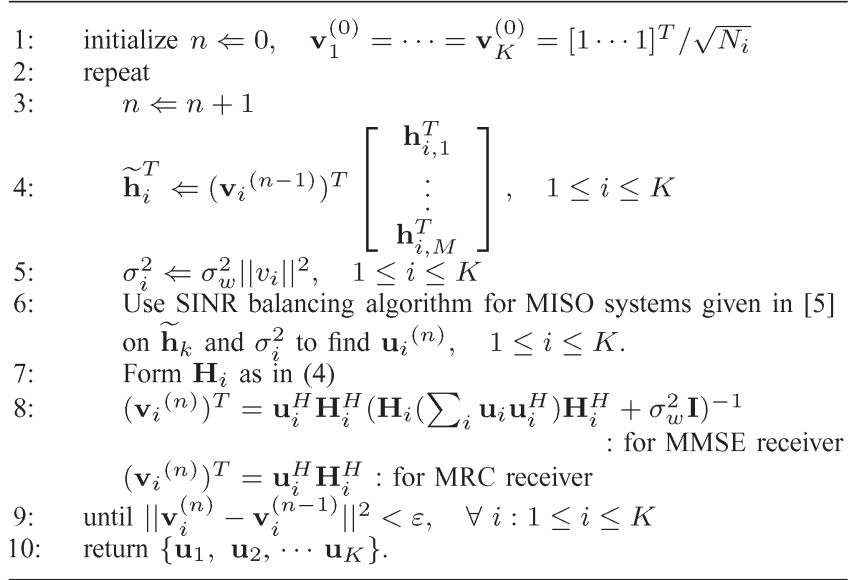

with the maximum eigenvalue. The optimal decoding vector for the second user, i.e., $\mathbf{v}_{2}$, can be also found similarly.

The optimal precoding and decoding vectors in the sense of maximizing the worst-case SINR can then be found by fixing one of the vectors and optimizing over the other one until convergence. We note that, when decoding vectors are fixed, the SINR balancing algorithm in [5] levels the SINR values of all users such that the worst SINR value is maximized. When precoding vectors are fixed, the SINR values of all users are increased by the selection of generalized eigenvectors, as discussed above. In other words, at every iteration cycle, the worstcase SINR value is increased. For this system, the convergence is guaranteed since the maximum SINR for all users is bounded from above due to nonzero $\sigma_{w}^{2}$ white noise at every receiver.

MRC Receivers: When MRC receivers are used instead of MMSE receivers, one may modify the proposed method by selecting $\mathbf{v}_{m}^{T}$ as $\mathbf{u}_{m}^{H} \mathbf{H}_{m}^{H}$ for user $m$, as shown in Table II. As can be seen from (16), this choice is not guaranteed to increase the SINR level of user $m$. Therefore, optimization for MRC receivers is not guaranteed to converge. In the following section, we examine the performance of SINR balancing for MMSE and MRC receivers. The implementation details of the SINR balancing algorithm are given in Table II.

\section{NUMERICAL RESULTS}

We have conducted computer simulations to illustrate the bit-errorrate (BER) performance of the proposed schemes. For the simulations, unit-energy quaternary phase-shift keying symbols are transmitted to $K$ users with $\left\{N_{1}, N_{2}, \ldots, N_{K}\right\}$ antennas through $N_{t}$ transmitting antennas. Each user receives one symbol per channel access, i.e., a single stream of data. We show such a system with the notation $N_{t} \times$ $\left(N_{1}, N_{2}, \ldots, N_{K}\right)$.

\section{A. Performance Comparison}

In Fig. 2, a transmitting system with three antennas serving three users, each with a single antenna, is compared with a MIMO system with three users having two antennas at each user. A $3 \times(1,1,1)$ system is simulated using the SINR balancing method [5], the decomposition method [3], and the joint-MMSE method [8]. The $3 \times(2,2,2)$ system is simulated using the methods that are proposed in this paper and the decomposition method that is given in [14], which uses MSE-optimized decoders after decomposition and the joint-MMSE method [8]. The methods of [5] and [3] are not applicable to the $3 \times(2,2,2)$ system due to the antenna limitations. In addition, 


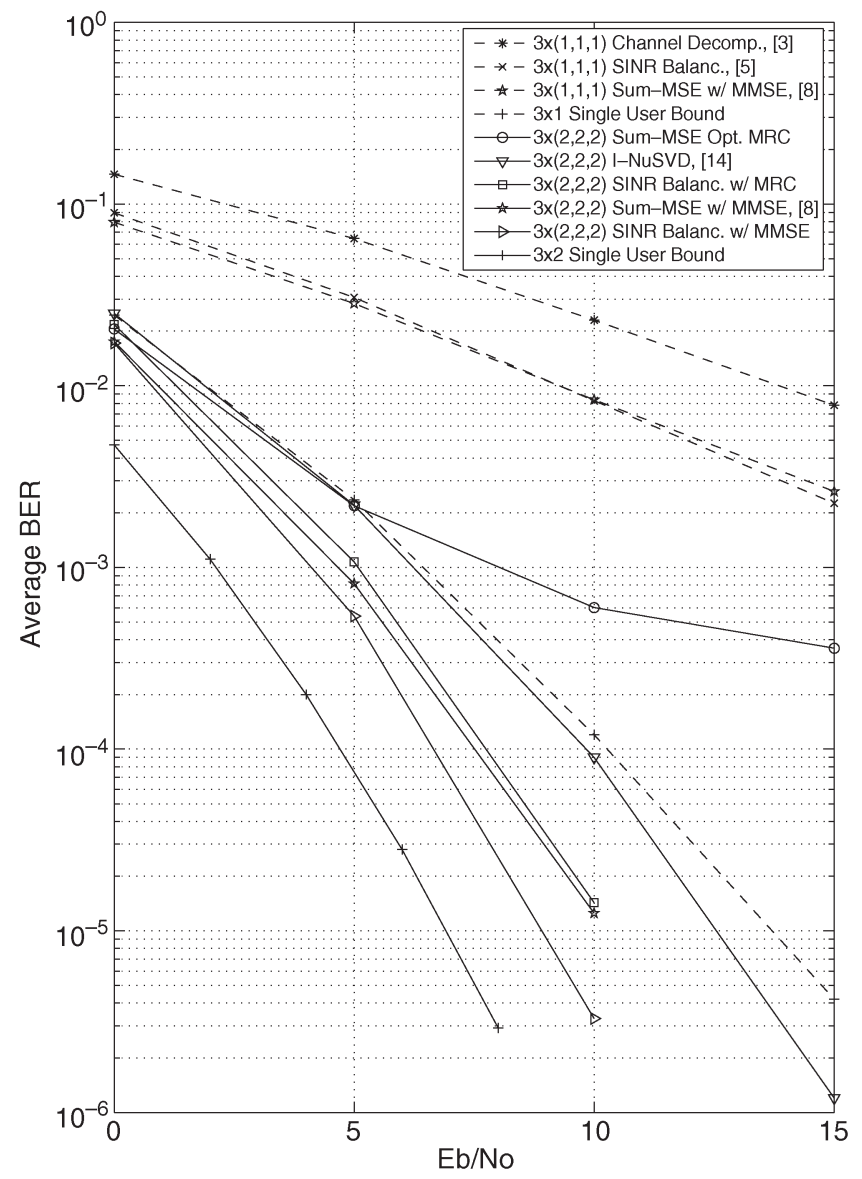

Fig. 2. Comparison of precoding methods for $3 \times(1,1,1)$ and $3 \times(2,2,2)$ systems.

the single-user bound for each system is also plotted for comparison purposes.

In Fig. 2, it is observed that the SINR balancing method with MRC receivers works as efficiently as the sum-MSE system with MMSE receivers. Furthermore, by comparing the slope of the SINR balancing graph with the single-user bound, we can say that the full diversity order, which is 6 , is realized by the proposed scheme. The value of a system upgrade from a $3 \times(1,1,1)$ system to a $3 \times(2,2,2)$ system with the SINR balancing method can be quantified as a gain of more than $10 \mathrm{~dB}$ at the BER value of $10^{-3}$.

Fig. 3 illustrates a communication system with more degrees of freedom. A transmitter having six antennas serves three users, each having a single antenna. The system is compared with a MIMO system where each user has two antennas. Again, the SINR balancing method with MRC receivers provides a gain almost identical to the gain of the sum-MSE system with MMSE receivers, which is a gain of $5 \mathrm{~dB}$ at a BER value of $10^{-5}$. The sum-MSE optimization framework has an error floor at high SNR values, indicating the lack of success at MAI mitigation.

\section{B. On the Convergence of Proposed Algorithms}

Sum-MSE and SINR balancing algorithms are guaranteed to converge with MMSE receivers. The convergence is due to the improvement in the objective criterion, i.e., a smaller MSE value or a larger SINR, at every update. When the receiver structure is fixed to MRC, the sum-MSE method still improves its objective at the precoder design stage, but a similar improvement is not guaranteed at the receiver design stage. It should be remembered that MRC is the optimum

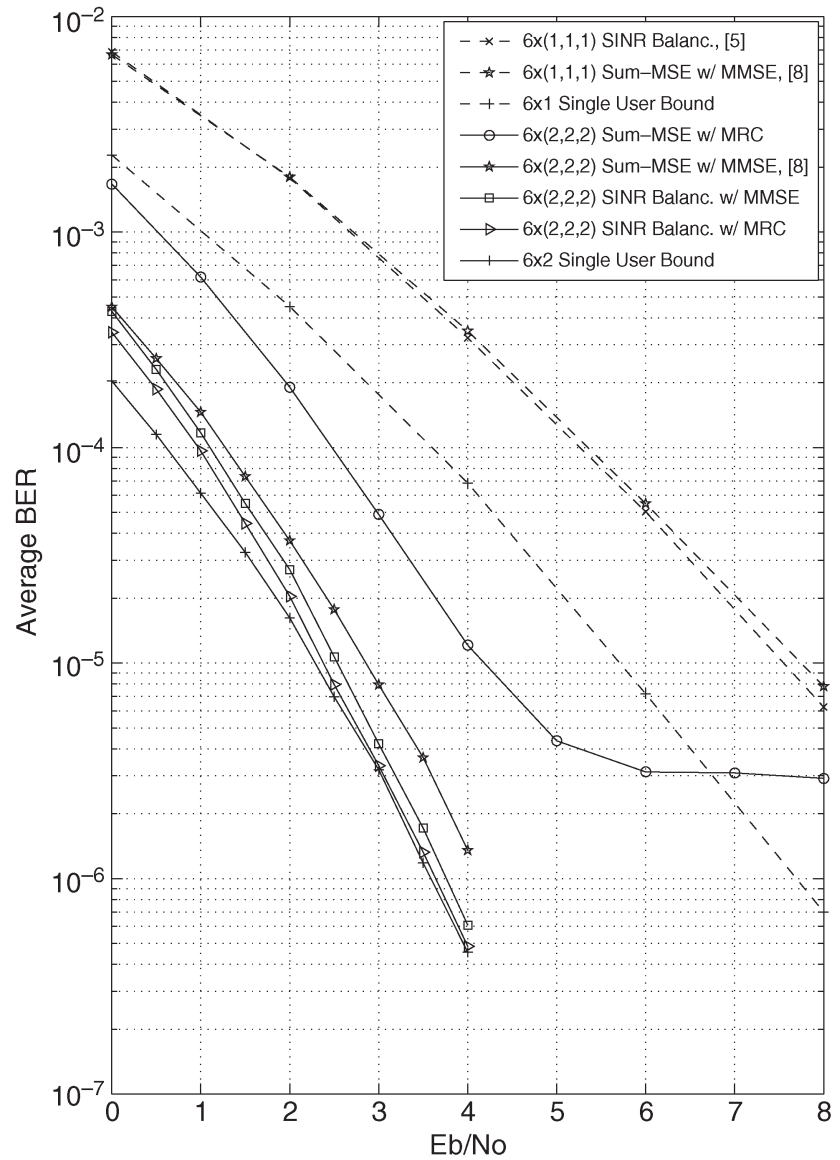

Fig. 3. Comparison of precoding methods for $6 \times(1,1,1)$ and $6 \times(2,2,2)$ systems.

receiver in the absence of MAI. Therefore, if MAI can be properly mitigated with a proper selection of precoders, MRC receivers can yield a performance similar to the joint-MMSE system.

The error floor for the sum-MSE framework with MRC receivers indicates that the precoders designed for MRC receivers are not as successful as the precoders of the joint-MMSE system at MAI mitigation. This is due to the attraction of the iterative algorithm to one of the local minima instead of progressing toward the global minimum of the cost function, as previously described.

Contrary to the sum-MSE framework, the SINR balancing framework with MRC receivers almost yields the performance of MMSE receivers. It should be remembered that, with the SINR balancing framework, the user with the lowest SINR value is guaranteed to improve at the next iteration cycle. With the suboptimal MRC receivers, the SINR of the user with the poorest MAI becomes the main optimization criterion. Therefore, the SINR balancing method keeps on improving the performance of the overall system, even with suboptimal MRC receivers. Table III presents the distribution of iterations required for the termination of MMSE- and MRC-based receivers for the SINR balancing framework. Table III is generated by averaging more than 100000 Monte Carlo runs at each SNR level. It can be observed that the convergence of algorithms is quite rapid for both systems. The convergence is faster for MRC receivers since the degrees of freedom for the optimization of MRC receivers are smaller than those of MMSE receivers.

Fig. 4 confirms the rapid convergence of MRC receivers in comparison with MMSE receivers. It can be seen from the top panel that a minor change in the termination criterion significantly affects the performance of MMSE systems, whereas a similar change does not 
TABLE III

Distribution OF THE NUMBER OF ITERATIONS FOR THE TERMINATION OF MMSE- AND MRC-BASED SINR BALANCING METHOdS FOR A $3 \times(2,2,2)$ SYSTEM

\begin{tabular}{|c|c||c|c|c||c|c|c||}
\hline & \multicolumn{3}{|c||}{} & \multicolumn{3}{c||}{ MMSE } & \multicolumn{3}{c||}{ MRC } \\
\hline$\varepsilon$ & SNR & Iteration \# [1-20] & Iteration \# [20-40] & Iteration \# > 40 & Iteration \# [1-20] & Iteration \# [20-40] & Iteration \# > 40 \\
\hline $1 \mathrm{e}-3$ & $0 \mathrm{~dB}$ & $89.13 \%$ & $10.05 \%$ & $0.82 \%$ & $99.34 \%$ & $0.62 \%$ & $0.04 \%$ \\
\hline $1 \mathrm{e}-3$ & $5 \mathrm{~dB}$ & $67.21 \%$ & $28.58 \%$ & $4.21 \%$ & $99.20 \%$ & $0.74 \%$ & $0.06 \%$ \\
\hline $1 \mathrm{e}-3$ & $10 \mathrm{~dB}$ & $52.63 \%$ & $36.91 \%$ & $10.46 \%$ & $98.48 \%$ & $1.39 \%$ & $0.13 \%$ \\
\hline $1 \mathrm{e}-4$ & $0 \mathrm{~dB}$ & $65.24 \%$ & $29.18 \%$ & $5.58 \%$ & $98.23 \%$ & $1.56 \%$ & $0.21 \%$ \\
\hline $1 \mathrm{e}-4$ & $5 \mathrm{~dB}$ & $18.39 \%$ & $52.55 \%$ & $29.06 \%$ & $98.05 \%$ & $1.71 \%$ & $0.24 \%$ \\
\hline $1 \mathrm{e}-4$ & $10 \mathrm{~dB}$ & $1.85 \%$ & $28.51 \%$ & $69.64 \%$ & $97.02 \%$ & $2.66 \%$ & $0.32 \%$ \\
\hline
\end{tabular}
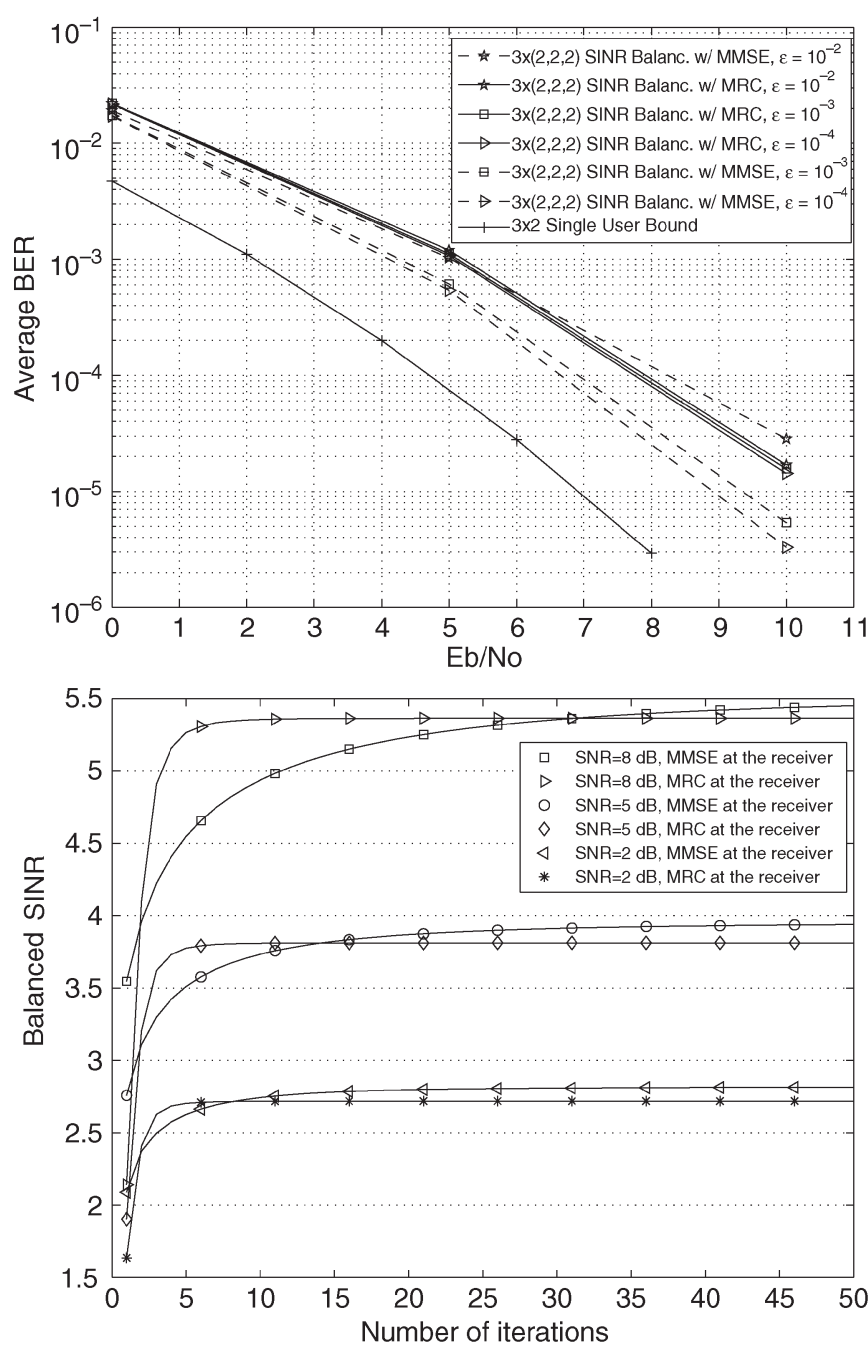

Fig. 4. Performance comparison of MRC- and MMSE-based SINR balancing methods for a $3 \times(2,2,2)$ system.

have an equivalent effect on MRC systems. The bottom panel shows the average SINR values with respect to the number of iterations for the system shown in the top panel. It can be noted from this figure that both MRC- and MMSE-based SINR balancing systems have almost the same SINR value at the algorithm termination, but MRC-based systems require fewer iterations for termination in comparison with MMSE systems. This can be explained by the multimodality of optimization space, in which MMSE systems are guaranteed to maximize the worst-case SINR by moving to a better point in the optimization space at every iteration. The MRC system has fewer degrees of freedom and cannot escape a local maximum. This leads to a poorer BER performance but a better convergence speed for MRC receivers.

\section{CONCLUSION}

We have presented two precoding methods that are suitable for multiuser MIMO systems having MRC receivers. Conventionally, MRC is not sufficient to remove MAI of multiuser systems, and MMSE receivers are utilized for interference suppression. In this paper, we have examined the utilization of precoding on multiuser systems having MRC receivers. It has been shown that a BER performance almost identical to the MMSE performance is realizable through the described SINR balancing framework with MRC receivers. This suggests that simple coherent receivers can perform as well as MMSE receivers if precoding vectors can be properly selected. The overall reduction in the hardware cost by the replacement of higher complexity receivers with MRC receivers can be particularly important for systems with many users.

\section{REFERENCES}

[1] D. Tse and P. Viswanath, Fundamentals of Wireless Communication. Cambridge, U.K.: Cambridge Univ. Press, 2005.

[2] M. Kang and M. S. Alouini, "A comparative study on the performance of MIMO MRC systems with and without cochannel interference," IEEE Trans. Commun., vol. 52, no. 8, pp. 1417-1425, Aug. 2004.

[3] L. U. Choi and R. D. Murch, "A transmit preprocessing technique for multiuser MIMO systems using a decomposition approach," IEEE Trans. Wireless Commun., vol. 3, no. 1, pp. 20-24, Jan. 2004.

[4] A. Shah and A. M. Haimovich, "Performance analysis of maximal ratio combining and comparison with optimum combining for mobile radio communications with cochannel interference," IEEE Trans. Veh. Technol., vol. 49, no. 4, pp. 1454-1463, Jul. 2000

[5] M. Schubert and H. Boche, "Solution of the multiuser downlink beamforming problem with individual SINR constraints," IEEE Trans. Veh. Technol., vol. 53, no. 1, pp. 18-28, Jan. 2004.

[6] Q. Spencer, A. Swindlehurst, and M. Haardt, "Zero-forcing methods for downlink spatial multiplexing in multiuser MIMO channels," IEEE Trans. Signal Process., vol. 52, no. 2, pp. 461-471, Feb. 2004.

[7] A. Khachan, A. Tenenbaum, and R. S. Adve, "Linear processing for the downlink in multiuser MIMO systems with multiple data streams," in Proc. IEEE Int. Conf. Commun., 2006, vol. 9, pp. 4113-4118.

[8] J. Zhang, Y. Wu, S. Zhou, and J. Wang, "Joint linear transmitter and receiver design for the downlink of multiuser MIMO systems," IEEE Commun. Lett., vol. 9, no. 11, pp. 991-993, Nov. 2005.

[9] C. Windpassinger, R. F. H. Fischer, T. Vencel, and J. B. Huber, "Precoding in multiantenna and multiuser communications," IEEE Trans. Wireless Commun., vol. 3, no. 4, pp. 1305-1316, Jul. 2004.

[10] J. Liu and W. A. Krzymien, "A novel nonlinear precoding algorithm for the downlink of multiple antenna multi-user systems," Wireless Pers. Commun., vol. 41, no. 2, pp. 207-223, Apr. 2007.

[11] S. Shi, M. Schubert, and H. Boche, "Downlink MMSE transceiver optimization for multiuser MIMO systems: MMSE balancing," IEEE Trans. Signal Process., vol. 56, no. 8, pp. 3702-3712, Aug. 2008.

[12] A. Wiesel, Y. C. Eldar, and S. Shamai, "Linear precoding via conic optimization for fixed MIMO receivers," IEEE Trans. Signal Process., vol. 54, no. 1, pp. 161-176, Jan. 2006.

[13] D. Reynolds, X. Wang, and K. N. Modi, "Interference suppression and diversity exploitation for multiantenna CDMA with ultra-low complexity receivers," IEEE Trans. Signal Process., vol. 53, no. 8, pp. 3226-3237, Aug. 2005. 
[14] Z. Pan, K. K. Wong, and T. S. Ng, "Generalized multiuser orthogonal space-division multiplexing," IEEE Trans. Wireless Commun., vol. 3, no. 6, pp. 1969-1973, Nov. 2004.

[15] A. Goldsmith, Wireless Communications. Cambridge, U.K.: Cambridge Univ. Press, 2005.

[16] L. U. Choi, "Multi-user MISO and MIMO transmit signal processing for wireless communication," Ph.D. dissertation, Hong Kong Univ. Sci. Technol., Hong Kong, 2003.

[17] A. Coskun, "Downlink transmission techniques for multi user multi input multi output wireless communications," M.S. thesis, Middle East Tech. Univ., Ankara, Turkey, 2007.

\section{A New Efficient Low-Complexity Scheme for Multi-Source Multi-Relay Cooperative Networks}

\author{
Haiyang Ding, Jianhua Ge, \\ Daniel Benevides da Costa, Member, IEEE, and Zhuoqin Jiang
}

\begin{abstract}
This paper proposes a new efficient scheme for the combined use of cooperative diversity and multiuser diversity. Considering a decodeand-forward (DF) opportunistic relaying strategy, we first study the outage behavior of the joint source-relay-selection scheme with/without direct links, from which the significance of the direct links is recognized. Motivated by the crucial role of these links on the system performance, a two-step selection scheme is proposed, which first selects the best source node based on the channel quality of the direct links and then selects the best link from the selected source to the destination. The proposed scheme significantly reduces the amount of channel estimation while achieving comparable outage performance to that using the joint selection scheme. Most importantly, the achieved diversity order is the same as that using the joint selection scheme. In addition, assuming an amplify-andforward (AF) opportunistic relaying strategy, the outage behavior of this two-step scheme is also analyzed, and its availability is confirmed as well.
\end{abstract}

Index Terms-Cooperative diversity, diversity order, multiuser diversity (MUD), outage behavior.

\section{INTRODUCTION}

Cooperative diversity has emerged as a powerful technique to exploit spatial diversity in wireless networks without the need for multiple antennas implemented at the terminals. In this case, it mitigates the detrimental effects of the fading by forming virtual antenna arrays

Manuscript received June 21, 2010; revised November 9, 2010 and November 21, 2010; accepted December 8, 2010. Date of publication December 20, 2010; date of current version February 18, 2011. This work was supported by the Program for Changiiang Scholars and Innovative Research Team in University under Grant IRT0852, by the Natural Science Foundation of Guangdong Province under Grant U0635003, by the National Major Specialized Project of Science and Technology of China under Grant 2009ZX03003-003/004, by the "111" project under Grant B08038, by the National Natural Science Foundation of China under Grant 61001128 and Grant 61001207, and by the Ceará Council of Scientific and Technological Development (FUNCAP) under Grant BP1-0031-00090.01.00/10. The review of this paper was coordinated by Dr. D. W. Matolak.

H. Ding is with the State Key Laboratory of Integrated Service Networks, Xidian University, Xi' an 710071, China, and also with the Xi' an Communication Institute, Xi'an 710106, China (e-mail: dinghy2003@hotmail.com).

J. Ge is with the State Key Laboratory of Integrated Service Networks, Xidian University, Xi' an 710071, China (e-mail: jhge@ xidian.edu.cn).

D. B. da Costa is with the Federal University of Ceara (UFC), Sobral, CE, Brazil (e-mail: danielbcosta@ieee.org).

Z. Jiang is with the Xi' an Communication Institute, Xi' an 710106, China (e-mail:zhqjiang@163.com).

Color versions of one or more of the figures in this paper are available online at http://ieeexplore.iee.org.

Digital Object Identifier 10.1109/TVT.2010.2100416
[1]. Among the cooperative strategies, opportunistic relay selection is an outage-optimal and low-complexity strategy [2]. This strategy can be implemented in a distributed manner and can reduce the power consumption of terminals.

Recently, multiuser diversity (MUD) has attracted significant attention in cooperative systems. In this case, diversity is attained through the help of relays in which, by letting only the user with the highest instantaneous signal-to-noise ratio (SNR) transmit at a given time, the MUD gain can be collected in the form of improved outage performance or increased total throughput [3]-[5]. Focusing on the capacity or throughput issues, the combination of MUD and cooperative diversity has been studied in [6] and [7]. In [8], the authors formulated a MUD-based cooperative framework with one relay and analyzed its asymptotic performance, from which the diversity order was attained for three cooperative strategies. The results of [8] were extended to the MUD-based cooperative networks with multiple relays in [9]. However, in [9], the authors assumed that a group of relays is preallocated for each source before the transmission, which simplifies the analysis and may not find applicability in practical systems. Very recently, assuming that the relays are shared by all the sources, Sun et al. [10] presented a combined use of cooperative diversity and MUD (i.e., joint source-relay selection scheme) and analyzed the total diversity order of the system with opportunistic amplify-and-forward (AF) relaying strategy. Nevertheless, the joint source-relay selection scheme proposed in [10] had the need for estimating the channel state information (CSI) of all the links in addition to find the best link (direct or dual-hop link) out of all the potential ones (i.e., $M(N+1)$ candidates for the network with $M$ sources and $N$ relays) in real time, which rendered the complexity extremely high for large values of $M$ and $N$.

In this paper, assuming a decode-and-forward (DF) opportunistic relaying strategy, the diversity order of the joint selection scheme is analyzed, from which the crucial role of the direct links in the endto-end performance is recognized. Inspired by this fact, we propose a two-step selection scheme. Specifically, the best source is first selected based on the channel quality of the direct links, and then, the relay with the maximum dual-hop end-to-end SNR from the selected source to destination is chosen to perform the two-phase transmission. Finally, the destination processes the received signals during the two-phase transmission by using selection combining so that the best link (direct or dual-hop link) from the selected source to the destination is chosen. This way, the complexity is considerably reduced while the same diversity order can be achieved with that presented in [10]. In addition, the outage performance of this two-step scheme is also examined, from which it is shown that it can achieve comparable performance with that utilizing the joint source-relay selection scheme. Furthermore, we also examine the outage behavior of the proposed scheme when the AF opportunistic relaying strategy is utilized. In this case, it is shown that the proposed scheme is also very effective.

\section{System Models}

For comparison purposes, in this section, we focus on the same scenario as that of [10]. Specifically, we consider a cooperative wireless network with $M$ source nodes $S_{m}(m=1,2, \ldots, M)$, one destination node $D$, and $N$ relays $R_{n}, n=1,2, \ldots, N$. All nodes are single-antenna devices and operate in a half-duplex mode. A timedivision multiple-access scheme is employed for orthogonal channel access, and the channels pertaining to each link undergo independent but not necessarily identically distributed (i.n.i.d.) Rayleigh flat fading.

In the following, assuming a proactive DF opportunistic relaying strategy [2], we first study the joint source-relay selection scheme. 